Research Article

\title{
Development and Integration of a CO Detection System Based on Wavelength Modulation Spectroscopy Using Near-Infrared DFB Laser
}

\author{
Bin Li $(\mathbb{D}$, Shuo-Cheng Zhang $\mathbb{D}$, and Yao-Dan Chi $\mathbb{D}$ \\ Institute of Electrical \& Computer Engineering, Jilin Jianzhu University, Changchun 130012, China \\ Correspondence should be addressed to Yao-Dan Chi; 2484670561@qq.com
}

Received 28 August 2017; Revised 18 October 2017; Accepted 24 October 2017; Published 10 January 2018

Academic Editor: Stephane Schilt

Copyright (C) 2018 Bin Li et al. This is an open access article distributed under the Creative Commons Attribution License, which permits unrestricted use, distribution, and reproduction in any medium, provided the original work is properly cited.

\begin{abstract}
A wavelength modulation spectroscopy- (WMS-) based gas sensing system was established to measure concentration of carbon monoxide (CO) in the range $0-100 \%$. The CO absorption line at $1563.06 \mathrm{~nm}$ was scanned with a tunable distributed feedback (DFB) laser, and two InGaAs photodiodes were applied to perform optic-electric conversion. Without using commercial instruments, essential electrical circuits were self-developed and integrated, including laser temperature controller, laser current driver, signal generator, and digital lock-in amplifier. The gas cell deployed in the system was fiber coupled with a total effective optical path length of $50 \mathrm{~cm}$. The second-order harmonic signal was extracted, and experiments of gas detection were carried out to investigate the performance of the sensor, including detection repeatability, detection accuracy, response time, and limit of detection (LoD). Experiment results show that the sensor is reliable and has acceptable probing performance. The maximum relative detection error is less than $3.8 \%$, suggesting good detection stability. Benefiting from the self-developed sensor, the whole $\mathrm{CO}$ detection system has small size, affordable expense, and application potential.
\end{abstract}

\section{Introduction}

Carbon monoxide, which is odorless and colorless, is an important indicator gas of preventing spontaneous combustion of coal seam [1-4]. In order to avoid disasters and guarantee safety production in coal mines, $\mathrm{CO}$ concentration monitoring must be performed effectively in real time. Among the numerous gas sensing techniques, optical methods have been studied worldwide in recent years due to its advantages including high sensitivity, fast response time, nonintrusive nature, wide sensing range, and long lifespan [5-7]. Tunable diode laser absorption spectroscopy (TDLAS) using lasers as light sources is a powerful and widely used technique for gas sensing applications including environmental monitoring, industrial process control, chemical analysis, and combustion diagnostics [8-12]. In order to achieve highly gas sensing ability, wavelength modulation spectroscopy technique is widely adopted to achieve high signal-to-noise ratio (SNR). This technique using harmonic detection is one of the most effective methods to suppress the background noises. Therefore, TDLAS-WMS technology has been widely studied and reported in the trace gas detection field [13-16].

Distributed feedback (DFB) laser has numerous advantages including compact size, high spectral resolution, and narrow line width $[17,18]$. By modulating DFB laser's driving current and working temperature, the center wavelength can be tuned across the absorption line of a target gas. Thus, DFB lasers coupled with optical fiber can be used as light sources in gas sensing systems. Generally, a typical WMS gas detection system using a DFB laser as light source can be divided into three parts: (1) DFB laser and its driving devices-in order to meet the sensing requirements, the laser's output must be precisely controlled. Thus, commercial devices including laser current driver, laser temperature controller, and signal generator are widely applied in such systems; (2) gas cell and optical structure-gas cell, as the container of target gas, is also coupled with optical parts to provide the path for laser beam; and (3) the signal detection part is the backend of a WMS system. Analog or digital 


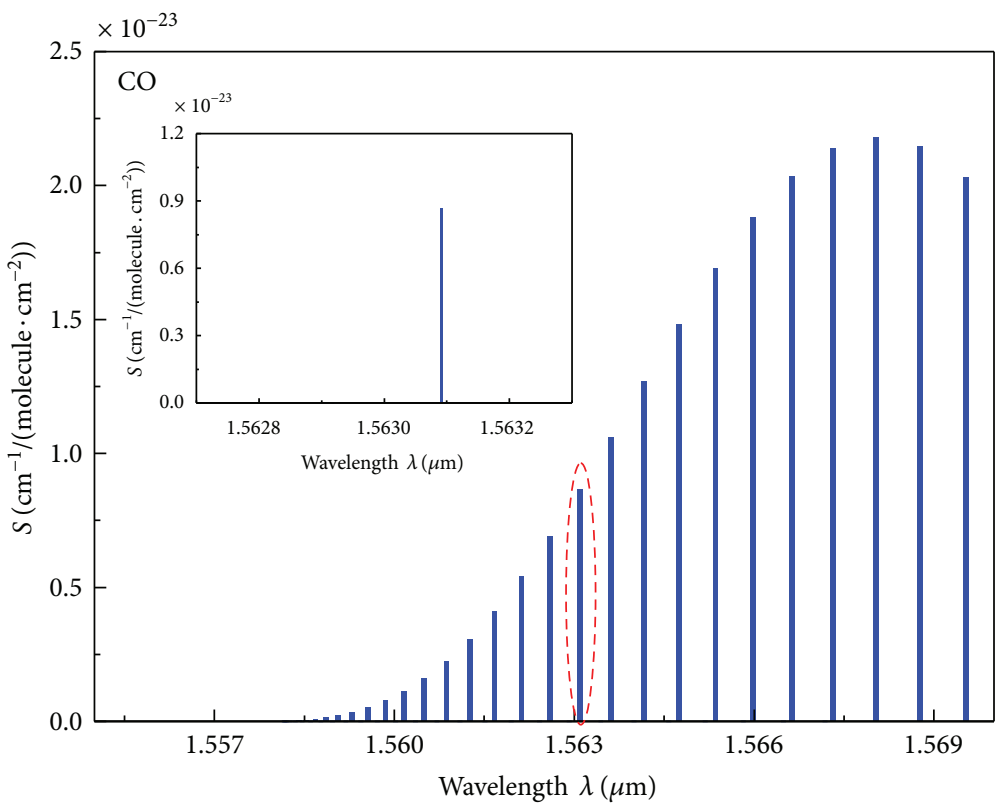

(a)

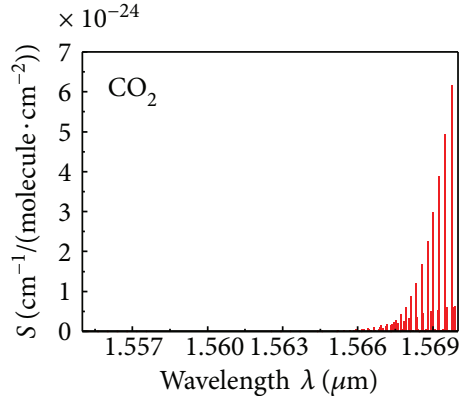

(b)

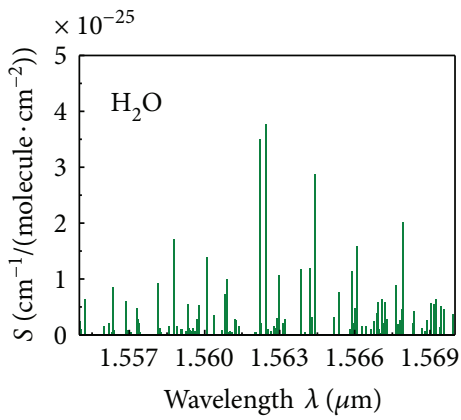

(c)

FIgURE 1: Absorption line of carbon monoxide (a), carbon dioxide (b), and $\mathrm{H}_{2} \mathrm{O}$ and target absorption line (c) in this gas detection system.

lock-in amplifiers are adopted to extract $1 \mathrm{f}$ and $2 \mathrm{f}$ harmonic signals which is related to gas concentration [19-22]. However, systems involving commercial instruments usually have some inescapable drawbacks including large size, high expense, and inconvenience to operate. These drawbacks are barriers for practical applications.

In view of the above considerations, a near-infrared $\mathrm{CO}$ sensing system based on the TDLAS-WMS technique was experimentally demonstrated. In this system, commercial instruments are replaced by self-developed circuits. A 14pin DFB laser with $1563.06 \mathrm{~nm}$ center wavelength is applied as the light source in this system. The laser's driving circuits and signal detection circuits were self-developed and integrated as a stand-alone instrument. Firstly, the gas sensing principle and system structure are introduced in this paper to guide the reader. Then, design details are shown with photos and descriptions. Finally, experiments were carried out and system performance was demonstrated.

\section{Structure and Theory}

2.1. CO Absorption Characteristics around $1.563 \mu \mathrm{m}$. The center wavelength of the DFB laser applied in this system is around $1.563 \mu \mathrm{m}$. The target $\mathrm{CO}$ absorption line in the near-infrared spectral region is shown in Figure 1(a). The absorption line strengths are obtained from HITRAN database [23]. CO detection around this band is affected very slightly by other major gases such as $\mathrm{CO}_{2}$ and $\mathrm{H}_{2} \mathrm{O}$. The absorption intensity of $\mathrm{CO}_{2}$ and $\mathrm{H}_{2} \mathrm{O}$ is shown in Figures 1(b) and 2(c). As shown in Figure 1(d), the target $\mathrm{CO}$ absorption line is clearly illustrated. In addition, another advantage of using a DFB laser in the near-infrared spectral region is that reliable and accessible telecommunication optical fiber can be applied for signal transmission in this system. Detailed optical signal transmission and reception will be described later in this paper.

2.2. System Structure. The schematic of this WMS-based CO detection system is shown in Figure 2. The whole system can be divided into four parts of hardware and their connections. The first part is the WMS-based sensor, which is the selfdeveloped instrument contents electrical circuit boards, DFB laser, fiber optic beam splitter (FOBS), optical attenuator (OA), InGaAs detectors, and input/output interfaces. The DFB laser, which is 14-pin butterfly-packaged, has a central wavelength of around $1.563 \mu \mathrm{m}$. A digital signal processor (DSP) chip (TMS320F28335) is applied as the main controller of this instrument. It controls the laser's temperature, driving current, and modulating signals by sending signals to corresponding circuits such as the TEC controlling circuit and DAC circuit. Meanwhile, it also processes signals which are related to gas concentration. Two InGaAs detectors are applied to convert the optical signal to electrical signal. The yellow lines in Figure 2 represent optical fibers. The second part of the system is a gas cell. Compared to our previous work [24], the effective optical absorption length has increased from $40 \mathrm{~cm}$ to $50 \mathrm{~cm}$ by utilizing a recently developed gas cell. The gas cell is fiber coupled and has a better gas tightness feature than our previous gas cells. Thirdly, a mass flow controller is applied in the system to perform dynamic gas mixing. It has a maximum $10 \mathrm{~L} / \mathrm{min}$ capability of distribution and three ports for connecting gas cylinders. These gas cylinders are the final part of the system and contain standard gases such as pure nitrogen and standard CO gas. The blue and red lines represent gas tubes and optical beam in Figure 2 . 


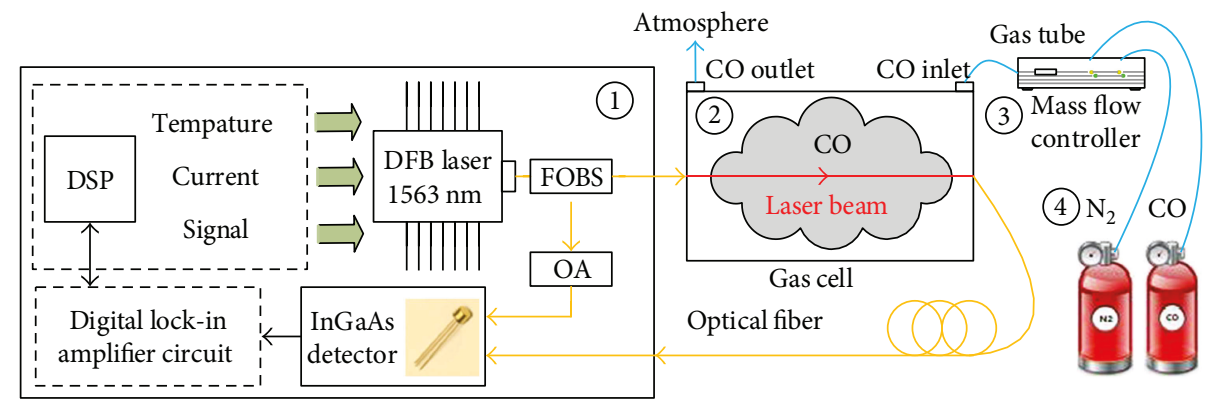

Figure 2: Schematic of the WMS-based CO detection system using a DFB laser at $1563 \mathrm{~nm}$.

2.3. Detection Theory. The fundamentals of the WMS technique have been reported widely by many research groups. In this section, only a brief background is described to familiarize the reader with various terms and explain certain experimental choices in this detection system. When the emitting wavelength of the DFB driver has been stabilized to the absorption peak of gas, a saw tooth wave signal $u_{\text {saw }}(t)=A_{\text {saw }}+\left(A_{\text {saw }} / T_{\text {saw }}\right)\left(t-T_{\text {saw }}\right)$ is generated to tune the central wavelength to scan the absorption line periodically. In this system, the frequency of the saw tooth signal is $10 \mathrm{~Hz}$. Meanwhile, in order to ensure the sensitivity and accuracy of detection, a sine signal $u_{\text {sin }}(t)=\left(A_{\sin } / 2\right)$ $\sin \left(\omega_{\sin } t\right)$ is generated to superimpose on the saw tooth signal. The driving signal of DFB laser can be expressed as $u(t)=u_{\text {saw }}(t)+u_{\text {sin }}(t)$. The below equation expresses the reference signal of light intensity:

$$
I_{r}(t)=n I_{0}[1+m u(t)]
$$

where $m, n$, and $I_{0}$ are the light modulation coefficient, the attenuation coefficient of $\mathrm{OA}$, and central emitting light intensity, respectively. According to the Beer-Lambert law, the absorption signal can be expressed below:

$$
\begin{aligned}
I_{t}(t) & =I_{0}[1+m u(t)] \exp [-\alpha(t) L C] \\
& \equiv I_{0}[1+m u(t)][1-\alpha(t) L C],
\end{aligned}
$$

where $\alpha(v, t)$ is the absorption coefficient dependent on the emitting wavelength of the DFB laser in the above equation. $L$ is the effective optical path length and $C$ is gas concentration. After optical-to-electrical conversion and amplification, signals from the gas cell channel and reference channel can be expressed below:

$$
\begin{aligned}
& u_{t}(t)=K_{t} D_{\mathrm{oe}}^{t} I_{0}[1+m u(t)][1-\alpha(t) L C] \\
& u_{r}(t)=K_{r} D_{\mathrm{oe}}^{r} n I_{0}[1+m u(t)],
\end{aligned}
$$

where $K_{t}, D_{\mathrm{oe}}^{t}, K_{r}$, and $D_{\mathrm{oe}}^{r}$ are the amplifying factor for the detection channel, optical-to-electrical conversion coefficient for the detection channel, amplifying factor for the reference channel, and optical-to-electrical conversion coefficient for the reference channel, respectively. Then, the differential signal of the absorption signal and the reference signal can be obtained below:

$$
\begin{aligned}
u(t) & =u_{r}(t)-u_{t}(t) \\
& =I_{0}[1+m u(t)]\left[K_{r} D_{\mathrm{oe}}^{r} n-K_{t} D_{\mathrm{oe}}^{t}+K_{t} D_{\mathrm{oe}}^{t} \alpha(t) L C\right] .
\end{aligned}
$$

By tuning $n$ of OA, let $K_{r} D_{\mathrm{oe}}^{r} n=K_{t} D_{\mathrm{oe}}^{t}$. Thus,

$$
u(t)=I_{0}[1+m u(t)]\left[K_{t} D_{\mathrm{oe}}^{t} \alpha(t) L C\right] .
$$

Considering that $m u(t)<<1$, the function below is obtained:

$$
u(t)=I_{0} K_{t} D_{\text {oe }}^{t} \alpha(t) L C .
$$

Extracting the second harmonic signal of $u(t)$,

$$
\begin{aligned}
S_{2}(\omega) & =\int_{t-T_{\sin }}^{t} u(\tau) e^{-j 2 \omega_{\sin } \tau} d \tau \\
& =\int_{t-T_{\sin }}^{t} I_{0} K_{t} D_{\mathrm{oe}}^{t} \alpha(\tau) L C e^{-j 2 \omega_{\sin } \tau} d \tau \\
& =K_{\mathrm{eq}} C \int_{t-T_{\mathrm{sin}}}^{t} \alpha(\tau) e^{-j 2 \omega_{\mathrm{sin}} \tau} d \tau,
\end{aligned}
$$

where $K_{\mathrm{eq}}=I_{0} K_{t} D_{\mathrm{oe}}^{t} L$ and $T_{\mathrm{sin}}=2 \pi / \omega_{\mathrm{sin}}$.

$S_{2}(\omega)$ is the intensity of the second harmonic signal on the frequency of $2 \omega_{\sin }$. The frequency of the saw signal and sine signal is set to be $T_{\text {saw }}=10 \mathrm{~Hz}$ and $T_{\text {sin }}=5 \mathrm{KHz}$, respectively. Then, an inverse Fourier transform is performed for the transformation from frequency domain to time domain. The $2 \mathrm{f}$ signal in each saw wave period can be obtained by the summation of a continuous second-order harmonic wave component in the time domain as shown in (8). The amplitude of the $2 \mathrm{f}$ signal, which is expressed as $\operatorname{Amp}\left[S_{2}(t)\right]$, can be obtained from the peak-to-peak value as shown in (9).

$$
\begin{aligned}
S_{2}(t) & =\sum_{t=0}^{T_{\text {saw }}} S_{2}(\omega), \\
\operatorname{Amp}\left[S_{2}(t)\right] & =\max \left[S_{2}(\omega)\right]-\min \left[S_{2}(\omega)\right] .
\end{aligned}
$$

In order to simplify the expression of the amplitude of the 2f signal, $A_{2}(\omega)$ and $A_{2}(\mathrm{t})$ are introduced in (10) and (11), respectively. By combining the equations from (7) to (12), 
the amplitude of the $2 \mathrm{f}$ signal can be obtained and simplified in the time domain as shown in (13).

$$
\begin{aligned}
A_{2}(\omega) & =\int_{t-T_{\text {sin }}}^{t} \alpha(\tau) e^{-j 2 \omega_{\text {sin }} \tau} d \tau, \\
A_{2}(t) & =\sum_{t=0}^{T_{\text {sav }}} A_{2}(\omega), \\
\operatorname{Amp}\left[A_{2}(t)\right] & =\max \left[A_{2}(\omega)\right]-\min \left[A_{2}(\omega)\right], \\
\operatorname{Amp}\left[S_{2}(t)\right] & =K_{\text {eq }} C \operatorname{Amp}\left[A_{2}(t)\right] .
\end{aligned}
$$

Therefore, the relation of the gas concentration and the amplitude of the $2 \mathrm{f}$ signal is obtained. Also, it can be seen that the $2 \mathrm{f}$ signal is determined by the gas concentration.

\section{Design Details of Key Modules}

3.1. DFB Laser and NIR Detector. The DFB laser applied in this system, which is a butterfly-packaged fiber-coupled laser, is provided by the Institute of Semiconductors, Chinese Academy of Sciences (ISCAS). The center wavelength of this laser is around $1.563 \mu \mathrm{m}$ which can be tuned by modulating the laser's temperature and injection current. The maximum optical output power is $10 \mathrm{~mW}$ when the operating temperature is set as $45.18^{\circ} \mathrm{C}$ and the operating current is set as $73.4 \mathrm{~mA}$. The laser's threshold current and slope efficiency are $21.8 \mathrm{~mA}$ and $0.19 \mathrm{~W} / \mathrm{A}$, respectively. The laser's temperature is determined by a thermoelectric cooler (TEC) module which is packaged inside the laser. The TEC module can be controlled to adjust the laser's working temperature in order to locate the center wavelength to the absorption line. In addition, there are two InGaAs photodiodes (J22-18IR500U) applied in this system for optical-electrical conversion. The peak responsivity is $0.9 \mathrm{~A} / \mathrm{W}$ and the diameter of the photosensitive area is $0.5 \mathrm{~mm}$. The two photodiodes are applied for the absorption channel and reference channel in order to obtain the differential signal. The differential channel structure was applied to obtain the differential signal between the absorption signal and the reference signal. The obtained differential signal is for $2 \mathrm{f}$ signal extraction. By using the differential channel structure, the fluctuation of the light source and intrinsic noise can be eliminate in order to achieve better sensing accuracy.

3.2. Power Supply Module. Normal switching power supplies cannot meet the requirement of the laser temperature control circuit because the power ripple is too high. In this way, a low-ripple linear power supply is developed. This linear power supply is indispensable for the temperature control module. Moreover, electrostatic discharge (ESD) protection and surge protection are also put into use to protect the DFBL and to guarantee system stability. The photo of the self-developed power supply is shown in Figure 3. The custom-made transformer is packaged in a black case. The power supply provides four channels of DC voltage which are $5 \mathrm{~V},-5 \mathrm{~V}, 12 \mathrm{~V}$, and $-12 \mathrm{~V}$.

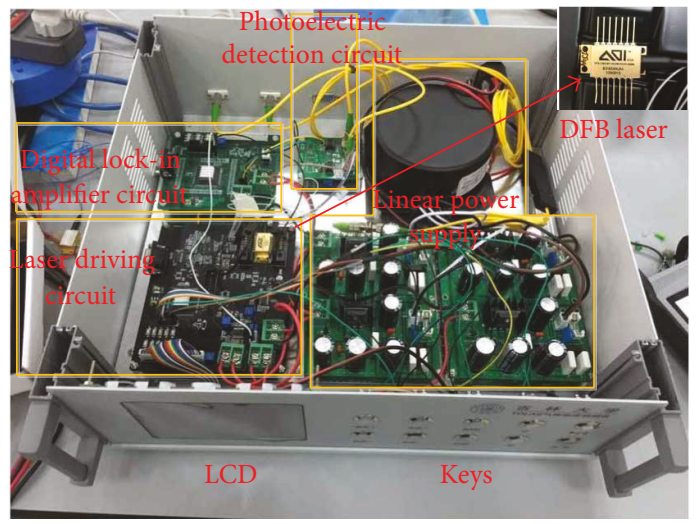

FIGURE 3: Inside view of the self-developed CO sensor based on the WMS technique.

3.3. Laser Driving Module. Temperature control for the DFB laser is necessary because the central wavelength depends on the laser's operation temperature while injection current is fixed. Thus, a temperature controller is developed to meet the requirements of control features including high stability, high accuracy, and quick response. Based on Peltier effect, the laser's temperature can be controlled by the TEC module packaged inside. The real-time temperature can be obtained by monitoring the inside-packaged thermistor with negative temperature coefficient (NTC). A high-efficiency and reliable monolithic TEC controller, ADN8831, is used to drive the TEC. It sets the temperature of the laser in a programmable way and maintains the temperature stable by using an analog PID control loop.

In this system, the temperature scope is set $35-55^{\circ} \mathrm{C}$ according to the laser test report. The center wavelength will be tuned around the absorption line when temperature is set to about $41.7^{\circ} \mathrm{C}$ while the injection current is $60 \mathrm{~mA}$. Experiments were carried out to investigate the performance of the self-developed temperature control circuit. As shown in Figure 4, the center wavelength is shifted linearly while the temperature is changed linearly from 39 to $43^{\circ} \mathrm{C}$ and the injection current is fixed as $80 \mathrm{~mA}$. This proves the good linearity of the temperature control circuit.

The V-I curve of the DFB laser applied in this system is provided in its test report. In this way, a DFB laser driving circuit is developed which is shown in Figure 3. The laser is mounted on a special socket which is soldered on the driving board. Under the condition of fixed temperature, the center wavelength is varied while changing the driving current. As shown in Figure 5, the laser temperature is fixed at $45^{\circ} \mathrm{C}$ and the driving current is increasing from 40 to $80 \mathrm{~mA}$. It can be observed that the center wavelength is shifted linearly. Meanwhile, the optical power is also increased observably.

A high-accuracy digital-to-analog converter (DAC, AD5060) chip is applied to set constant voltage or periodical changing voltage. In $\mathrm{CO}$ detection experiments, the laser temperature is set at first to locate the center wavelength at the absorption line. Then, a saw tooth wave signal at $10 \mathrm{~Hz}$ generated by the DAC will act on the laser's output in order to make the center wavelength scan across the absorption line periodically. The DAC's output is controlled by DSP's 


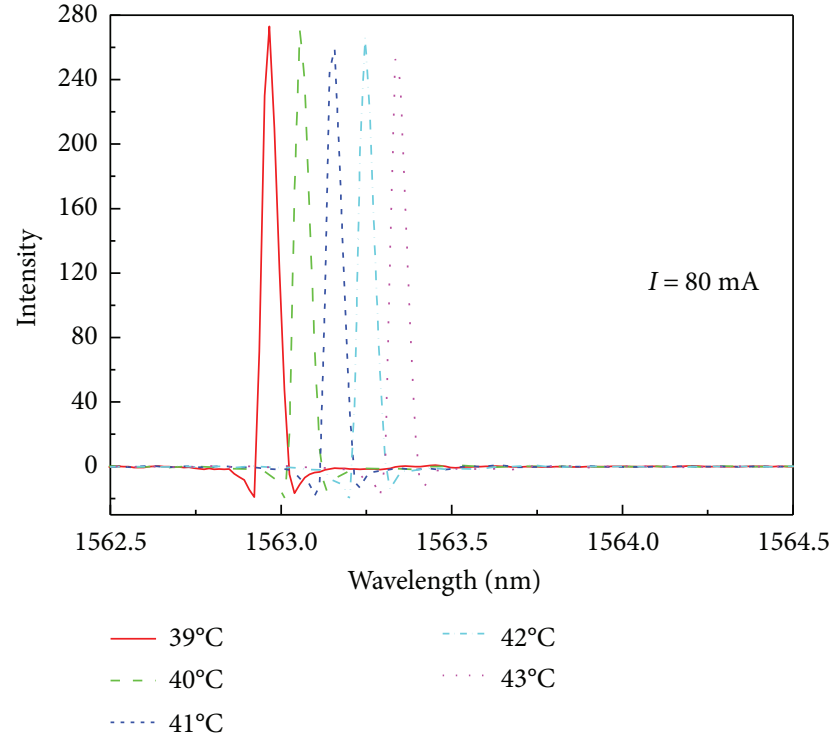

FIGURE 4: Center wavelength shifts while laser temperature is tuned linearly under constant injection current.

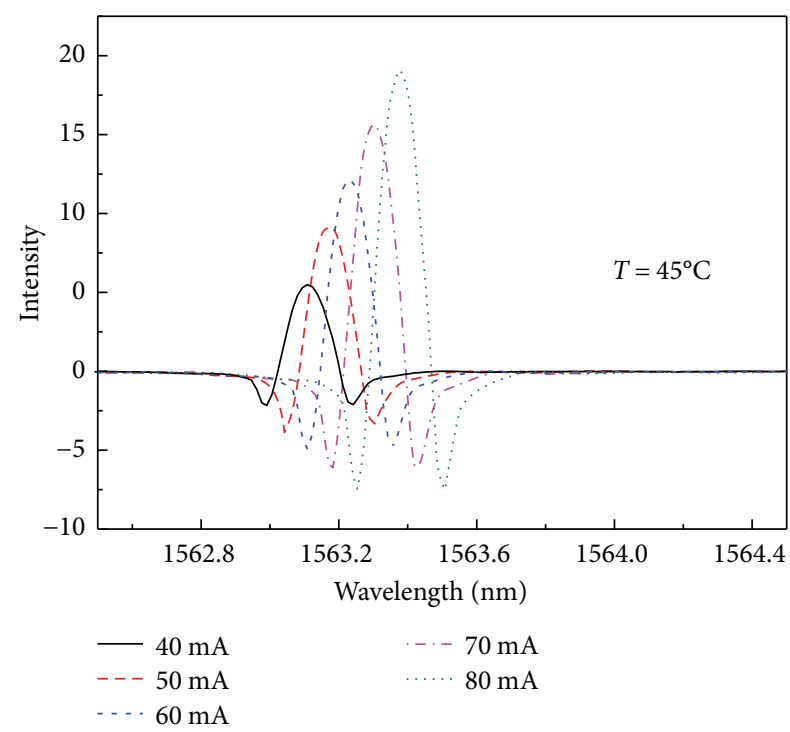

FIGURE 5: Under constant laser temperature, the center wavelength and optical power are controlled by injection current.

CPU timer. In WMS, there is also a sine wave signal required to modulate the laser's output. In this system, this is performed by a direct digital synthesizer (DDS, AD9851). Then, the saw tooth signal and sine wave signal are combined by AD8513, which is a precision and low-noise JFET amplifier.

3.4. Digital Lock-In Amplifier. In WMS, harmonic signals such as $1 \mathrm{f}$ and $2 \mathrm{f}$ are usually extracted by using commercial lock-in amplifiers. In this system, a digital lock-in amplifier based on DSP is developed and the photo is shown in Figure 3 . The $2 \mathrm{f}$ harmonic signal is isolated by the digital lock-in amplifier to indicated CO concentration. Experiments were carried out to evaluate its performance. As shown

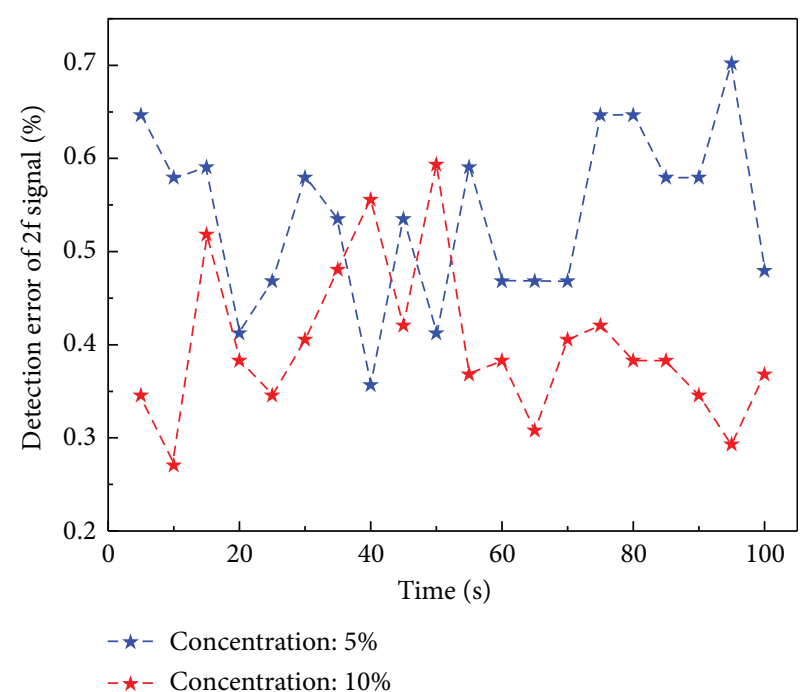

Figure 6: Performance test of the digital lock-in amplifier.

in Figure 6, under the concentration of $5 \%$ and $10 \%$ of carbon monoxide, the detection errors of the $2 \mathrm{f}$ signal are shown in blue and red data, respectively. It can be seen that the detection error is measured 20 times in a period of 100 seconds and the maximum error is under $0.7 \%$ which suggests good stability.

3.5. Gas Cell. According to the Beer-Lambert law, effective absorption optical path length $L$ is a very important parameter in a TDLAS system. This is due to the fact that the incensement of $L$ can lead to clearer absorption phenomenon and lower detection limit. Thus, the gas cell was redesigned and developed in this system. Compared to our previous work, the path length is increased from $40 \mathrm{~cm}$ to $50 \mathrm{~cm}$. This gas cell is integrated with a pair of optical fiber collimator. The gas cell is a single-pass cell. It is stable and suitable for gas sensing experiments.

\section{Experiments and Discussion}

4.1. Experimental Setup. The actual experimental setup is shown in Figure 7. The DFB laser and essential selfdeveloped circuits are integrated in a stand-alone instrument as the CO sensor. The details of this sensor is described in the last section, and the inside of the instrument is shown in Figure 3. The gas cell, which has a total effective optical path length of $50 \mathrm{~cm}$, is connected with the CO sensor and the mass flow controller by the optical fiber and air tube, respectively. The sample gas for concentration measurements was supplied from a combination of two channels of the mass flow controller. One is connected with pure $\mathrm{N}_{2}$ and the other is connected with the standard $\mathrm{CO}$ gas. For the measurements, a total flow of $1 \mathrm{~L} / \mathrm{min}$ was used. There are manual knobs on the front panel of the mass flow controller for flow control. Each time the mass flow controller is powered on, there is an initializing process of the mass flow controller to achieve better accuracy and this period of time lasts about 15 minutes. 


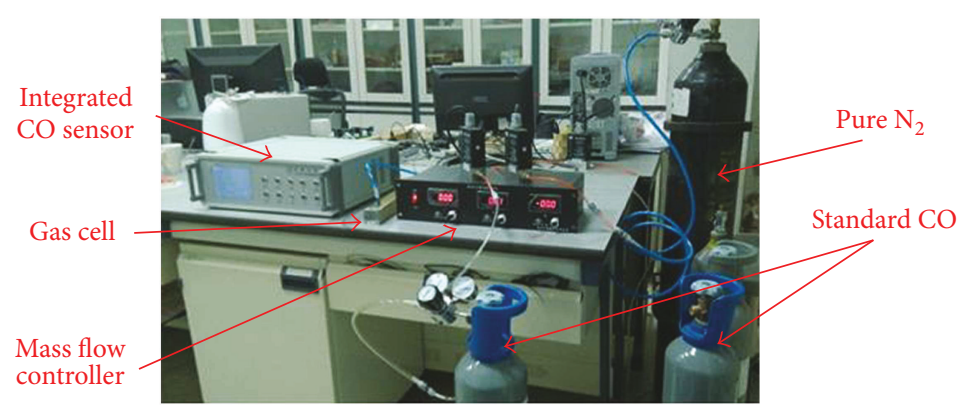

FIgURE 7: Laboratory experimental setup of the WMS-based CO sensor.

TABLE 1: Results of the detection repeatability test under $70 \%$ concentration.

\begin{tabular}{ccccccc}
\hline$N$ & 1 & 2 & 3 & 4 & 5 & 6 \\
\hline Ai & $70.56 \%$ & $70.41 \%$ & $71.12 \%$ & $70.06 \%$ & $69.88 \%$ & $70.25 \%$ \\
\hline
\end{tabular}

During the CO detection experiments, the DFB laser temperature was set to $42^{\circ} \mathrm{C}$ to locate the center wavelength at the absorption line. The laser driving current is shifting in the range of $40-80 \mathrm{~mA}$, and the frequency is $10 \mathrm{~Hz}$. The $5 \mathrm{kHz}$ sine wave signal is generated to be the modulation signal. These operations can be done by pressing the keys embedded on the front panel of the instrument. These experimental conditions were kept constant for $\mathrm{CO}$ concentration measurement carried out for performance evaluation which are discussed below.

4.2. Detection Repeatability. Experiments were carried out to investigate the repeatability of the performance firstly. Pure $\mathrm{N}_{2}$ was injected into the gas cell, and the concentration was set to zero of the CO sensor (instrument) while the gas is filled full of $\mathrm{N}_{2}$. Then, a concentration of $70 \% \mathrm{CO}$ gas was filled into the gas cell and the results were recorded. The whole process was repeated six times (as shown in Table 1) and the results were recorded for analysis. The repeatability $S_{\mathrm{r}}$ is calculated by using the equation below:

$$
S_{\mathrm{r}}=\frac{1}{\bar{A}} \sqrt{\frac{\sum_{i=1}^{n}\left(A_{i}-\bar{A}\right)^{2}}{n-1}} \times 100 \%
$$

where $A_{i}$ is the concentration result displayed on the LCD, $n$ is the number of measured times, $n=6, \bar{A}$ is the arithmetic average of results, and $S_{\mathrm{r}}$ is the detection repeatability. Thus, $S_{\mathrm{r}}=1.38 \%$ was calculated and it satisfies the project's requirement of $S_{\mathrm{r}}<2 \%$.

4.3. Response Time. Distinguished with chemical gas sensors, infrared optical sensors do not require time for chemical reaction and the sensing process is in real time. However, there is still a short period of time consumed by signal transmitting, data calculation, delays in program code, and LCD refreshing rate. Besides, it is not convenient to read the display on LCD if data is varying too fast. In this way, the display cycle is set as 2 seconds in this system.

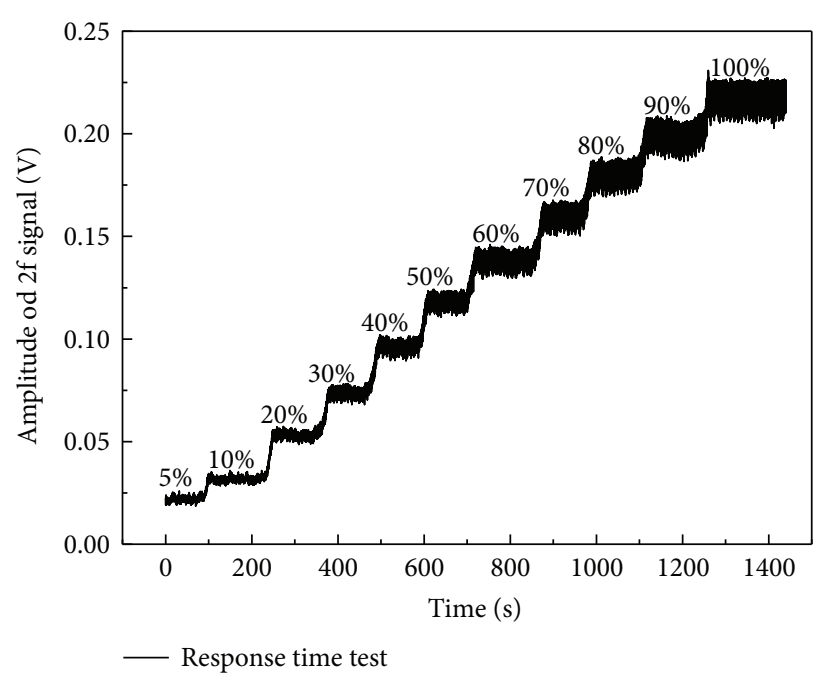

FIgURe 8: Response time test of the system under $1 \mathrm{~L} / \mathrm{min}$ flow speed.

Due to the advantage of quick response, the sensor can be used in fast-changing applications. For example, experiments were performed to acquire the variation of concentration in the gas cell and the result is shown in Figure 8. By changing the $\mathrm{CO}$ concentration from $5 \%$ to full scale, the $2 \mathrm{f}$ signal was recorded. As shown in Figure 8, there are slopes between adjacent concentrations. These slopes were corresponding to time which was consumed by manual operations and gas injecting to the gas cell. Therefore, compared to traditional gas sensors, the developed sensor can be utilized to applications demanding quick response.

4.4. Detection Accuracy. Experiments were carried out to investigate the detection accuracy. Measured concentrations as well as the standard concentrations of $10 \mathrm{CO}$ samples are comparatively shown in Figure 9(a). The relative error of each gas sample was calculated, as shown in Figure 9(b). Under the concentration of $10 \times 10^{4} \mathrm{ppm}$, the relative error becomes the maximum, which is about $3.8 \%$. As shown in Figure 10, the absolute error increases while the concentration raises. However, with the increasing of concentration, the relative error tends to be decreasing and within the whole detection range, the relative detection error is within the range of $-2.9 \% \sim 3.8 \%$. This confirms that the developed system reveals acceptable performances. 


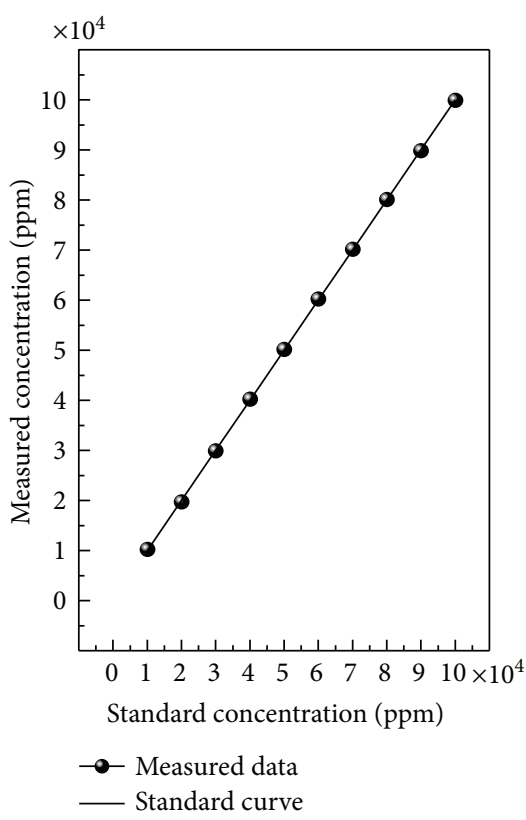

(a)

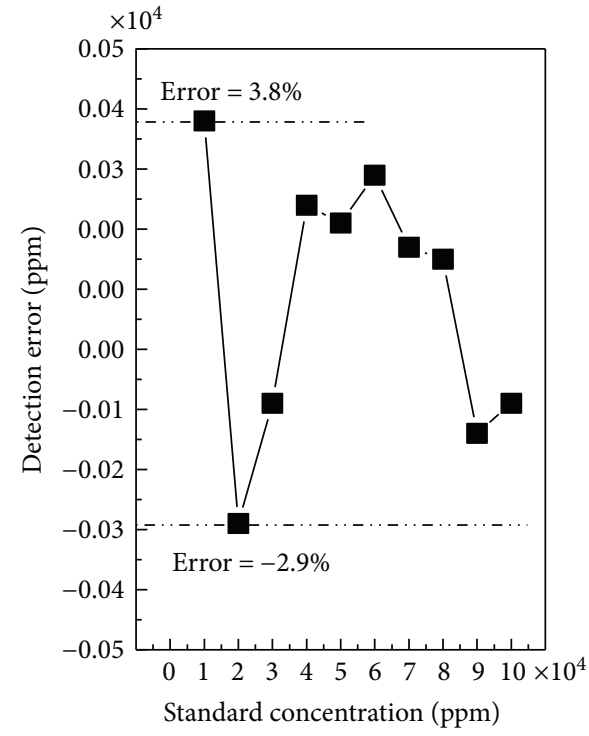

(b)

Figure 9: (a) Measured concentrations as well as standard concentrations and (b) relative detection error test.

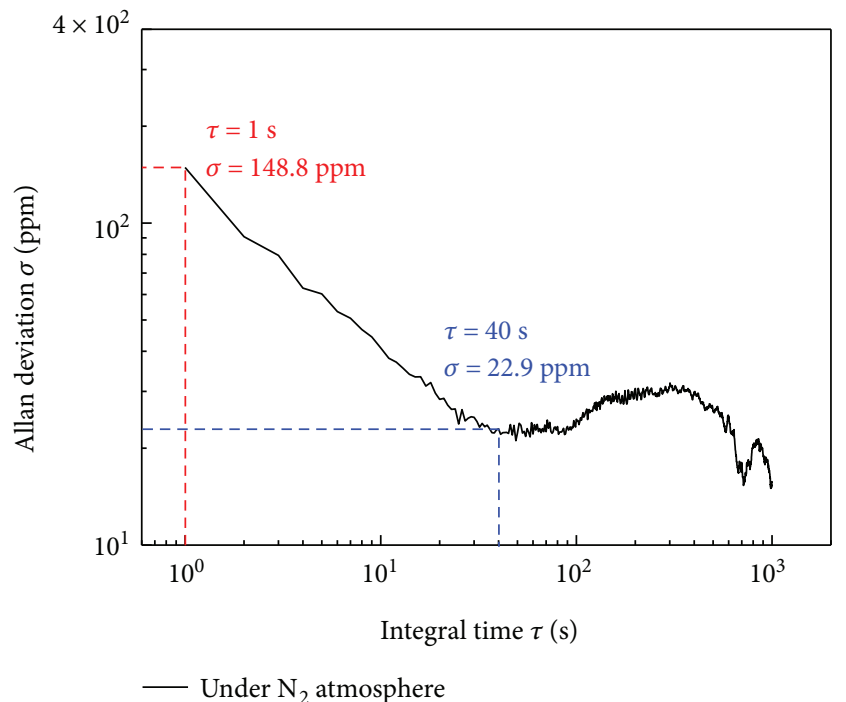

Figure 10: The Allan deviation curve of the detection system.

4.5. Limit of Detection. The Allan deviation curve of the detection system is shown in Figure 10, where the integral time is within the range of $1-1000 \mathrm{~s}$. With $1 \mathrm{~s}$ integral time, the Allan deviation value of the system is about $148.8 \mathrm{ppm}$. The system shows the best stability with an integral time of around $40 \mathrm{~s}$, where the corresponding Allan deviation value is about $22.9 \mathrm{ppm}$.

\section{Conclusion}

Based on the TDLAS technique, a CO detection system was experimentally demonstrated. Without using commercial instruments, the self-developed electrical part includes a linear power supply module, a laser temperature control and modulation module, and a reliable digital lock-in amplifier. Under the operation of the developed DFB laser driver, the laser temperature can be controlled effectively. The laser's emitting wavelength varies linearly with the temperature and injection current. The developed digital lock-in amplifier can effectively extract the harmonic signals, and the maximum extraction errors on the harmonic amplitude for two gas samples are less than $1 \%$ for $2 \mathrm{f}$. As the CO concentration increases from $10 \%$ to $100 \%$, the amplitudes of the $2 \mathrm{f}$ harmonic signals were obtained. The limit of detection is estimated to be about $22.9 \mathrm{ppm}$, and the relative detection error is within the range of $-2.9 \% \sim 3.8 \%$. The whole CO detection system has high sensitivity, small size, affordable expense, and application potential.

\section{Conflicts of Interest}

The authors declare that they have no conflicts of interest.

\section{Acknowledgments}

The authors wish to express their gratitude to Dr. Xiaotian Yang, National Natural Science Foundation of China (no. 51672103), Jilin Jianzhu University, the National Key Technology R\&D Program of the Ministry of Science and Technology of China, the Education Department of Jilin Province of China, and the State Key Laboratory of Integrated Optoelectronics, Jilin University for their generous support of this work. 


\section{References}

[1] A. A. I. Khalil, M. A. Gondal, and N. Al-Suliman, "Resonant photo-acoustic detection of carbon monoxide with UV Laser at $213 \mathrm{~nm}, "$ Applied Physics B: Lasers and Optics, vol. 103, no. 2, pp. 441-450, 2011.

[2] X. H. Li, X. M. Liu, Y. K. Gong, H. B. Sun, L. R. Wang, and K. Q. Lu, “A novel erbium/ytterbium co-doped distributed feedback fiber laser with single-polarization and unidirectional output," Laser Physics Letters, vol. 7, no. 1, pp. 55-59, 2010.

[3] Y. X. Meng, T. G. Liu, K. Liu et al., "A modified empirical mode decomposition algorithm in TDLAS for gas detection," IEEE Photonics Journal, vol. 6, no. 6, pp. 1-7, 2014.

[4] J. Vingerhoets, J. Snoeys, and S. Minten, "Detection of smouldering fires by carbon monoxide gas concentration measurement," Chemical Engineering Transactions, vol. 48, p. 457, 2016.

[5] H. Moser, A. Genner, J. Ofner, C. Schwarzer, G. Strasser, and B. Lendl, "Application of a ring cavity surface emitting quantum cascade laser (RCSE-QCL) on the measurement of $\mathrm{H}_{2} \mathrm{~S}$ in a $\mathrm{CH}_{4}$ matrix for process analytics," Optics Express, vol. 24, no. 6, pp. 6572-6585, 2016.

[6] R. Ghorbani and F. M. Schmidt, "Real-time breath gas analysis of $\mathrm{CO}$ and $\mathrm{CO}_{2}$ using an EC-QCL," Applied Physics B: Lasers and Optics, vol. 123, no. 5, p. 144, 2017.

[7] G. D. Banik, S. Som, A. Maity et al., "An EC-QCL based $\mathrm{N}_{2} \mathrm{O}$ sensor at $5.2 \mu \mathrm{m}$ using cavity ring-down spectroscopy for environmental applications," Analytical Methods, vol. 9, no. 15, pp. 2315-2320, 2017.

[8] G. Zhang, J. Liu, Z. Xu, Y. He, and R. Kan, "Characterization of temperature non-uniformity over a premixed $\mathrm{CH}_{4}$-air flame based on line-of-sight TDLAS," Applied Physics B: Lasers and Optics, vol. 122, no. 1, p. 3, 2016.

[9] B. Buchholz, S. Kallweit, and V. Ebert, "SEALDH-II-an autonomous, holistically controlled, first principles TDLAS hygrometer for field and airborne applications: designsetup-accuracy/stability stress test," Sensors, vol. 17, no. 1, p. $68,2017$.

[10] D. Choi, M. Jeon, G. Cho, T. Kamimoto, Y. Deguchi, and D. Doh, "Performance improvements in temperature reconstructions of 2-D tunable diode laser absorption spectroscopy (TDLAS)," Journal of Thermal Science, vol. 25, no. 1, pp. 8489, 2016.

[11] Y. Krishna and S. Obyrne, "Tunable diode laser absorption spectroscopy as a flow diagnostic tool: a review," Journal of the Indian Institute of Science, vol. 96, p. 17, 2016.

[12] X. Zhou, J. Yu, L. Wang, Q. Gao, and Z. Zhang, "Sensitive detection of oxygen using a diffused integrating cavity as a gas absorption cell," Sensors and Actuators B: Chemical, vol. 241, pp. 1076-1081, 2017.

[13] A. Upadhyay, D. Wilson, M. Lengden, A. L. Chakraborty, G. Stewart, and W. Johnstone, "Calibration-free WMS using a cw-DFB-QCL, a VCSEL, and an edge-emitting DFB laser with in-situ real-time laser parameter characterization," IEEE Photonics Journal, vol. 9, no. 2, pp. 1-17, 2017.

[14] W. Wei, J. Chang, Y. Y. Liu et al., "Eliminating the effect of phase shift between injection current and amplitude modulation in DFB-LD WMS for high-precision measurement," Applied Optics, vol. 55, no. 13, pp. 3526-3530, 2016.

[15] S. S. Choi, A. Mandelis, X. Guo, B. Lashkari, S. Kellnberger, and V. Ntziachristos, "Wavelength-modulated differential photoacoustic spectroscopy (WM-DPAS) for noninvasive early cancer detection and tissue hypoxia monitoring," Journal of Biophotonics, vol. 9, no. 4, pp. 388-395, 2016.

[16] Z. Du, J. Li, X. Cao, H. Gao, and Y. Ma, "High-sensitive carbon disulfide sensor using wavelength modulation spectroscopy in the mid-infrared fingerprint region," Sensors and Actuators B: Chemical, vol. 247, pp. 384-391, 2017.

[17] H. Virtanen, T. Uusitalo, and M. Dumitrescu, "Simulation studies of DFB laser longitudinal structures for narrow linewidth emission," Optical and Quantum Electronics, vol. 49, no. 4, p. 160, 2017.

[18] T. Yang, Y. Song, W. Zhang, and F. Li, "Acoustic emission detection using intensity-modulated DFB fiber laser sensor," Chinese Optics Letters, vol. 14, no. 12, article 120602, 2016.

[19] L. M. Song, L. W. Liu, Y. G. Yang, Q. H. Guo, and J. T. Xi, “An optical sensor for hydrogen sulfide detection in open path using WMS-2f/1 $f$ technique," Optoelectronics Letters, vol. 12, no. 6, pp. 465-468, 2016.

[20] S. Neethu, R. Verma, S. Kamble, J. K. Radhakrishnan, P. P. Krishnapur, and V. C. Padaki, "Validation of wavelength modulation spectroscopy techniques for oxygen concentration measurement," Sensors and Actuators B: Chemical, vol. 192, pp. 70-76, 2014.

[21] Q. Wang, J. Chang, F. P. Wang, and W. Wei, "Recovery of pure wavelength modulation second harmonic signal waveforms in distributed feedback diode laser-based photoacoustic spectroscopy," Sensors and Actuators A: Physical, vol. 245, pp. 54-62, 2016.

[22] T. Benoy, M. Lengden, G. Stewart, and W. Johnstone, "Recovery of absorption line shapes with correction for the wavelength modulation characteristics of DFB lasers," IEEE Photonics Journal, vol. 8, pp. 1-17, 2016.

[23] L. S. Rothman, D. Jacquemart, A. Barbe et al., "The HITRAN 2004 molecular spectroscopic database," Journal of Quantitative Spectroscopy and Radiative Transfer, vol. 96, pp. 139204, 2003.

[24] B. Li, C. T. Zheng, H. F. Liu et al., "Development and measurement of a near-infrared $\mathrm{CH} 4$ detection system using $1.654 \mu \mathrm{m}$ wavelength-modulated diode laser and open reflective gas sensing probe," Sensors and Actuators B: Chemical, vol. 225, pp. 188-198, 2016. 

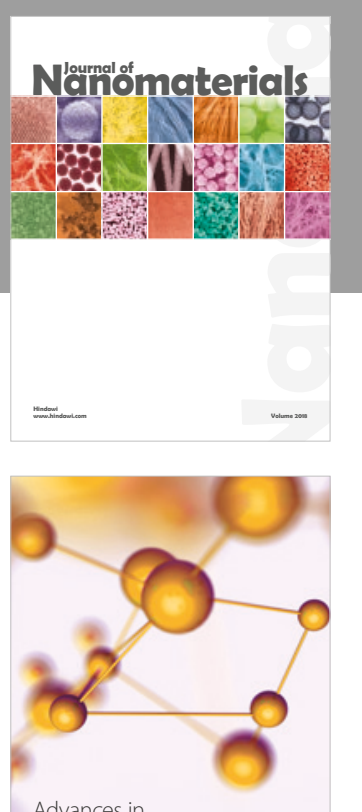

Physical Chemistry
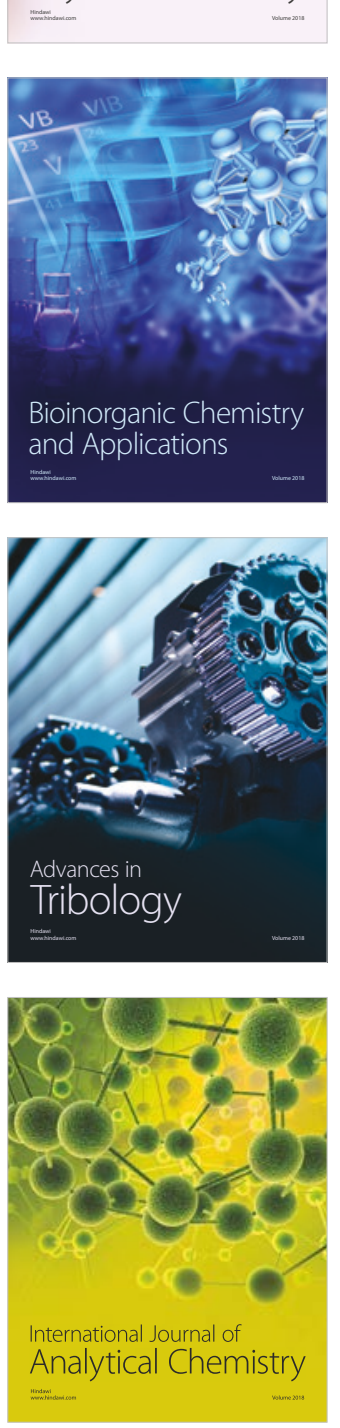

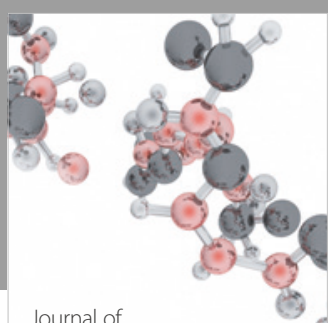

Analytical Methods

in Chemistry

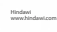

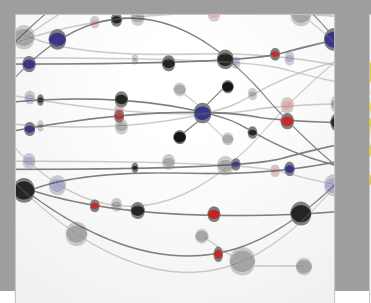

The Scientific World Journal

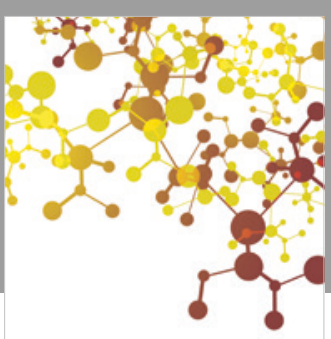

Journal of

Applied Chemistry
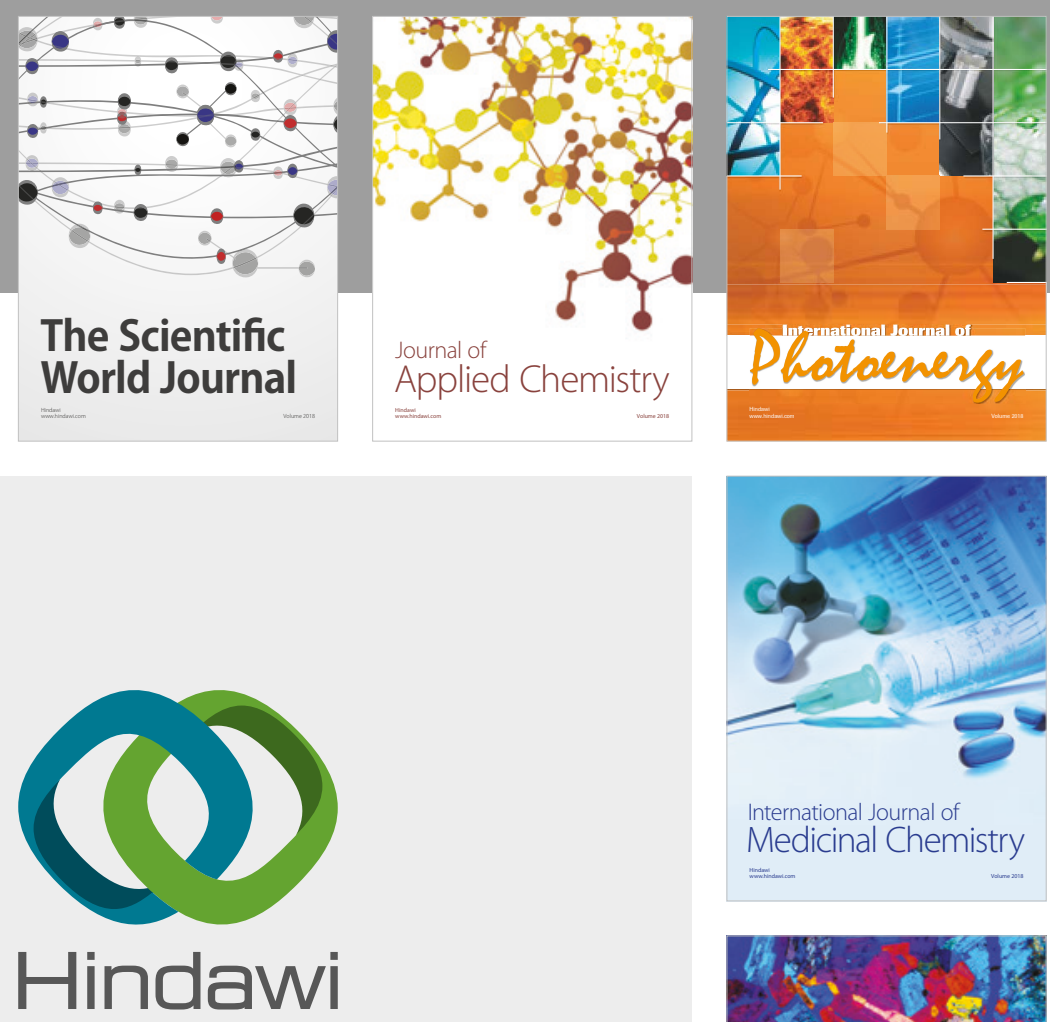

Submit your manuscripts at

www.hindawi.com
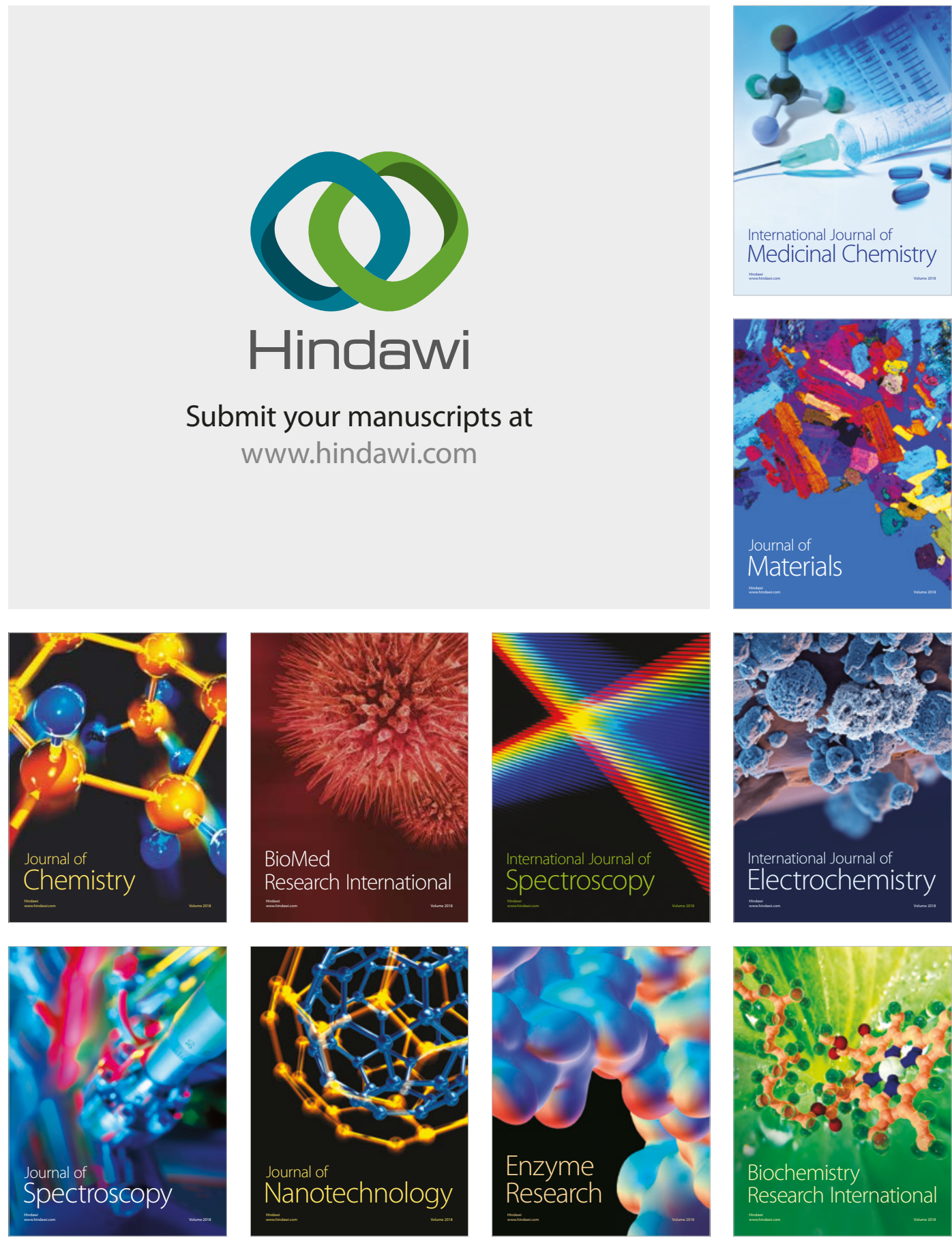
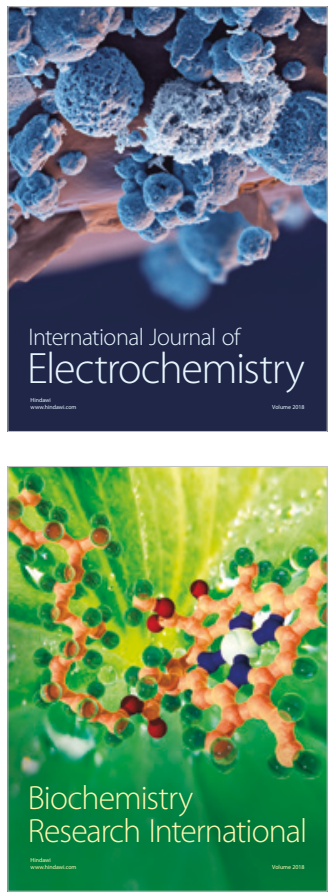